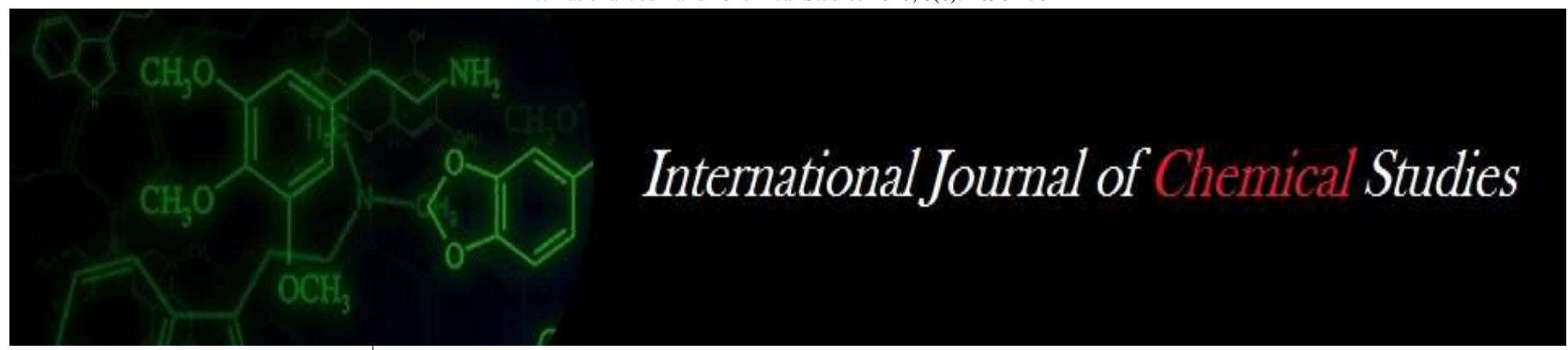

P-ISSN: 2349-8528

E-ISSN: 2321-4902

www.chemijournal.com

IJCS 2020; 8(6): 1696-1701

(C) 2020 IJCS

Received: 21-09-2020

Accepted: 06-11-2020

\section{PV Abhilash}

Department of Genetics and

Plant Breeding, Sam

Higginbottom University of

Agriculture \& Technology, Naini

Agriculture Institute, Prayagraj,

Uttar Pradesh, India

\section{B Sirisha Rani}

Department of Genetics and

Plant Breeding, Sam

Higginbottom University of

Agriculture \& Technology, Naini

Agriculture Institute, Prayagraj,

Uttar Pradesh, India

\section{Suresh BG}

Department of Genetics and

Plant Breeding, Sam

Higginbottom University of

Agriculture \& Technology, Naini

Agriculture Institute, Prayagraj,

Uttar Pradesh India

\section{B Jalandhar Ram}

Department of Genetics and

Plant Breeding, Sam

Higginbottom University of

Agriculture \& Technology, Naini Agriculture Institute, Prayagraj,

Uttar Pradesh India
Corresponding Author: PV Abhilash

Department of Genetics and Plant Breeding, Sam

Higginbottom University of Agriculture \& Technology, Naini Agriculture Institute, Prayagraj, Uttar Pradesh India

\section{Correlation and path analysis studies in finger millet for yield and yield contributing traits [Eluesine coracana (L.) Gaertn.]}

\author{
PV Abhilash, B Sirisha Rani, Suresh BG and B Jalandhar Ram
}

DOI: https://doi.org/10.22271/chemi.2020.v8.i6x.11013

\begin{abstract}
The present investigation consists of 34 finger millet genotypes used for studying genetic variability parameters, correlation and path analysis which was carried out at Field Experiment Centre, Department of Genetics and Plant Breeding during Kharif 2019 in Randomized Block Design with three replications. The data were recorded for 15 quantitative characters to study genetic variability, heritability, genetic advance, correlation and path analysis. The analysis of variance indicated significant difference among 34 genotypes for all the characters studied. Moderate GCV and PCV values were observed for harvest index and test weight. High heritability coupled with high genetic advance as percent mean observed for plant height the role of additive genes in the inheritance of these traits and hence these characters could be improved through simple phenotypic selection. Correlation studies revealed significant positive association of grain yield per plant with harvest index for both genotypic level and phenotypic level. The path coefficient analysis indicated that selection for ear head length, number of fingers per ear, biological yield, harvest index as both genotypic level and phenotypic level would directly increase seed yield. IE3473 , IE-4759 were observed as promising genotypes for important quantitative traits.
\end{abstract}

Keywords: Finger millet, GCV, PCV, variability, heritability, correlation and path analysis

\section{Introduction}

Finger millet (Eleusine coracna (L.) Gaertn.) is one of the most important crop belonging to family and sub family Chloridoide. Finger millet is self-pollinated tetraploid $(2 n=36, A A B B)$. Based on morphological, cytological and molecular evidence, it is believed that modern finger millet (Eleusine coracana subsp. coracana) is domesticated from wild finger millet (Eleusine coracana subsp. africana) populations (chennaveeraiah and Hiremanth, 1974 and Hilu and Dewet, 1976) ${ }^{[6,11]}$. It can stored for long period up years or more deteriorating or weevil damage so it is excellent for storage time of famine Among with quantitative achievement in food production, important since cereal grain occupy a dominant part in the poor people's diet, contribution 70 percent of calories in most case in most along with significant amountof protein in finger millet and other nutrients. Finger millet is almost $99 \%$ self-pollinating with negligible 1\% cross pollination mediated by wind (Jansen and Ong, 1966, Purseglove, 1972) $[13,18]$. The estimation account further indicates the requirement for the encouragement of HYV to meet the aggressive globlal marketing setting. In order to achieve the expected targets there is need to develop the varieties with higher yield potential by the existing yield plateau utilizing more distant lines in the breeding programmes (Singamsetti et al., 2018) ${ }^{[2]}$

\section{Materials and methods}

The experiment was conducted to evaluate 34 genotypes of finger millet which were grown in Randomized Block Design (RBD) with three replications in Kharif -2019. The experimental field was divided into 3 blocks of equal size and each line possesses single genotype. The data were recorded on 15 quantitative characters viz., Days to $50 \%$ flowering, Days to maturity, Plant height $(\mathrm{cm})$, Number of leafs, Flag leaf length $(\mathrm{cm})$, Flag leaf width $(\mathrm{cm})$, Ear head length $(\mathrm{cm})$, No of fingers per plant, Finger length $(\mathrm{cm})$, Finger width $(\mathrm{cm})$, No of productive tillers, Biological yield(g), Harvest index, Test weight $(\mathrm{g})$, Grain weight $(\mathrm{g})$ per plant. Mean values were computed and data were analysed for analysis of variance as suggested Fisher (1936) given in table 1. Phenotypic coefficient of variation (PCV) and genotypic coefficient of 
variation (GCV) were given by Burton (1952) ${ }^{[3]}$. Heritability in broad sense was given by Lush (1949) ${ }^{[15]}$ and Burton and Devane (1953) ${ }^{[4]}$. Genetic advance was given by Lush (1949) ${ }^{[15]}$ and Johnson et al., (1955) ${ }^{[12]}$. Correlation coefficients are estimated as suggested by Al-Jibouri et al., (1958) ${ }^{[1]}$ and the path analysis was calculated as suggested by Dewey and Lu $(1959)^{[8]}$

\section{Results and discussion}

The analysis of variance carried out 15 quantitative characters revealed significant differences (Table 1). Hence it was concluded that there is scope for ample genetic variation among genotype under study and further statistical analysis was carried out. On the basis of mean performance, the highest grain yield per plant was observed for the finger millet genotype IE-3473 followed by IE-4759.

The PCV was higher than GCV for all the characters under study which indicated that the environment factors influencing the characters studied. Moderate GCV was recorded for harvest index (18.56), test weight (16.94), biological yield per plant (15.58), number of productive tillers (15.58), ear head length (12.20), number of leafs (11.13), plant height (10.385). Low GCV was recorded for finger length (9.50), grain yield per plant (8.62), flag leaf width (8.48), number of fingers per ear (8.41), finger width (7.95), flag leaf length (7.59), days to maturity (7.74).

Phenotypic coefficient of variation (PCV) ranged from days to maturity (7.92) to harvest index (19.83). Moderate PCV was recorded for number of harvest index (19.83), test weight (19.63), number of productive tillers (18.82), biological yield (16.00), flag leaf width (15.76), ear head length (15.37), number of leafs (14.56), flag leaf length (13.35), plant height (13.29), days to $50 \%$ flowering (12.95), finger width (12.26), finger length (11.47) and low PCV for number of finger per ear (9.66), days to maturity (7.92).

The estimation of heritability (\%) in the broad sense for 15 characters studied which range from flag leaf width (29.00) to days to $50 \%$ flowering (97.00). High heritability was recorded for days to $50 \%$ percent flowering(97.00), days to maturity (95.00), biological yield per plant (94.90), harvest index (87.70), number of fingers per ear (75.80), finger length (68.70), grain yield per plant (67.80), test weight (66.10), ear head length (63.00), plant height (61.00). Moderate heritability was recorded for number of leafs (58.580), number of productive tillers (51.90), finger width (42.00), flag leaf length (32.40). Low heritability was recorded for flag leaf width (29.00).

Genetic advance as \% of mean varied from flag leaf width (9.40) to harvest index (35.81). High genetic advance was recorded for harvest index (35.81), biological yield per plant (31.28), test weight (28.38), days to $50 \%$ flowering (25.89), number of productive tillers (20.120). Moderate genetic advance was recorded for ear head length (19.94), number of leafs (17.536), plant height (16.71), finger length (16.22), days to maturity (15.59), grain yield per plant (14.63), finger width (10.61). Low genetic advance was recorded for flag leaf width (9.40), flag leaf length (8.90).

Genotypic correlation between grain yield per plant showed positive significant genotypic association with harvest index Similar results are observed by Chavan et al. (2020) ${ }^{[5]}$ for harvest index. Phenotypic correlation between grain yield per plant showed positive significance phenotypic association with harvest index. Similar results are observed by Negi et al. (2017) ${ }^{[17]}$, Chavan et al. (2020) ${ }^{[5]}$ for harvest index.

In the present study the results of path coefficient analysis indicated that selection for ear head length, number of fingers per ear, biological yield, harvest index as both genotypic level and phenotypic level. Whereas days to $50 \%$ flowering, plant height, finger width, number of productive tillers, harvest index, test weight at genotypic level and days to maturity, number of leafs, flag leaf length, flag leaf width, finger length, harvest index at phenotypic level would directly increase seed yield. An increase in any one of these or all of this quantitative character would bring simultaneous increase in yield.

\section{Conclusion}

From the present investigation it is concluded that analysis of variance showed significant variation to all the characters. Among 34 genotypes of finger millet on the basis of mean performance IE-3473 (3.32g) shows maximum grain yield followed by IE-4759 (3.19g), IE-2568 (3.12g).

The estimates of GCV and PCV revealed that phenotypic coefficient of variation was higher than the genotypic coefficient of variation, which indicate presence of environment effect on expression on character studied. Moderate difference between GCV and PCV were depicted for test weight, harvest index.

Correlation studies revealed significant positive association of grain yield per plant with harvest index for both genotypic level and phenotypic level.

Path analysis revealed that the characters ear head length, number of fingers per ear, biological yield, harvest index as both genotypic level and phenotypic level. Hence utmost importance should be given to these characters during selection for seed yield per plant.

Application of research: Since the population is increasing there is urgent need to provide high yield varieties to meet the demand. Unavailability of cultivars with high potential. Therefore present study has been undertaken to identify the best hybrid which can give high yield.

Research Category: Genetics and Plant Breeding.

Acknowledgement/Funding: Authors are thankful to department of Genetics and Plant Breeding, Sam Higginbottom University of Agriculture Technology and Sciences, Prayagraj, Uttar Pradesh, India

Table 1: Analysis of variance for 15 characters of 34 finger millet genotypes during kharif-2019

\begin{tabular}{|c|c|c|c|}
\hline Source of Variations & Replicate & Treatments & Error \\
\hline DF & 2 & 33 & 66 \\
\hline Days to 50\% flowering & $10.42 *$ & $253.23 * *$ & 2.59 \\
\hline Days to maturity & $10.16 *$ & $184.91 * *$ & 2.83 \\
\hline Plant height (cm) & 77.34 & $520.23 * *$ & 91.32 \\
\hline No. Of leaves & 2.89 & $8.626 *$ & 1.65 \\
\hline Flag leaf length (cm) & 72.12 & $44.61 *$ & $18.32 * *$ \\
\hline Flag leaf width (cm) & 0.016 & $0.17 * *$ & 0.008 \\
\hline
\end{tabular}




\begin{tabular}{|c|c|c|c|}
\hline Ear head length (cm) & 0.010 & $2.47^{* *}$ & 0.406 \\
\hline Finger length (cm) & 0.42 & $0.93^{* *}$ & 0.123 \\
\hline Finger width (cm) & 0.002 & $0.008^{* *}$ & 0.002 \\
\hline No. of finger per ear & 0.066 & $0.6 * *$ & 0.58 \\
\hline No. of productive tillers & 0.007 & $0.15^{* *}$ & 0.035 \\
\hline Biological yield per plant $(\mathrm{g})$ & 0.152 & $34.85 * *$ & 0.614 \\
\hline Harvest index (\%) & 1.172 & $17.14 * *$ & 0.769 \\
\hline Test weight $(\mathrm{g})$ & 0.003 & $0.039^{* *}$ & 0.006 \\
\hline Grain yield per plant $(\mathrm{g})$ & 0.033 & $0.181 * *$ & 0.025 \\
\hline
\end{tabular}

** Significant at $1 \%$ Level of Significance, * Significant at 5\% Level of Significance

Table 2: Estimation of genetic parameters for grain yield and other components

\begin{tabular}{|c|c|c|c|c|c|}
\hline Genetic parameters Summary & GCV & PCV & $\mathbf{h}^{2}$ (Broad Sense) & Genetic Advancement 5\% & Gen.Adv as \% of Mean 5\% \\
\hline Days to 50\% flowering & 12.76 & 12.95 & 97.00 & 18.54 & 25.89 \\
\hline Days to maturity & 7.74 & 7.92 & 95.50 & 15.68 & 15.59 \\
\hline Plant height(cm) & 10.38 & 13.29 & 61.00 & 21.24 & 16.71 \\
\hline No. Of leaves & 11.13 & 14.56 & 58.50 & 2.40 & 17.53 \\
\hline Flag leaf length & 7.59 & 13.35 & 32.40 & 3.46 & 8.90 \\
\hline Flag leaf width(cm) & 8.48 & 15.76 & 29.00 & 0.062 & 9.40 \\
\hline Ear head length(cm) & 12.20 & 15.37 & 63.00 & 1.358 & 19.94 \\
\hline Finger length(cm) & 9.50 & 11.47 & 68.70 & 0.888 & 16.22 \\
\hline Finger width(cm) & 7.95 & 12.26 & 42.00 & 0.056 & 10.61 \\
\hline No. of finger per ear & 8.41 & 9.66 & 75.80 & 0.762 & 15.08 \\
\hline No. of productive tillers & 13.56 & 18.82 & 51.90 & 0.290 & 20.12 \\
\hline Biological yield per plant $(\mathrm{g})$ & 15.58 & 16.00 & 94.901 & 6.77 & 31.28 \\
\hline Harvest index (\%) & 18.56 & 19.83 & 87.70 & 4.506 & 35.81 \\
\hline Test weight(g) & 16.94 & 19.63 & 66.10 & 0.17 & 28.38 \\
\hline Grain yield per plant(g) & 8.62 & 10.47 & 67.80 & 0.387 & 14.63 \\
\hline
\end{tabular}

$\mathrm{GCV}=$ Genotypic coefficient of variation, $\mathrm{PCV}=$ Phenotypic coefficient of variation,

$\mathrm{GA}=$ Genetic

Table 3: Genotypic correlation coefficient between yield and its related traits in 15 quantitative parameters in finger millet

\begin{tabular}{|c|c|c|c|c|c|c|c|c|c|c|c|c|c|c|c|}
\hline characters & $\begin{array}{c}\text { Days to } \\
50 \% \\
\text { flowering }\end{array}$ & $\begin{array}{c}\text { Days to } \\
\text { maturity }\end{array}$ & $\mid \begin{array}{c}\text { Plant } \\
\text { height } \\
(\mathbf{c m})\end{array}$ & $\begin{array}{l}\text { No of } \\
\text { leafs }\end{array}$ & $\begin{array}{c}\begin{array}{c}\text { Flag } \\
\text { leaf } \\
\text { length } \\
(\mathrm{cm})\end{array} \\
\end{array}$ & $\begin{array}{c}\text { Flag leaf } \\
\text { width } \\
(\mathrm{cm})\end{array}$ & $\begin{array}{c}\text { Ear head } \\
\text { length } \\
\text { (cm) }\end{array}$ & $\begin{array}{c}\text { Finger } \\
\text { length } \\
(\mathbf{c m})\end{array}$ & $\begin{array}{c}\text { Finger } \\
\text { width } \\
(\mathrm{cm})\end{array}$ & $\begin{array}{c}\text { No of } \\
\text { fingers } \\
\text { per ear }\end{array}$ & $\begin{array}{c}\text { No of } \\
\text { productiv } \\
\text { e tillers }\end{array}$ & $\begin{array}{c}\text { Biologica } \\
\text { l yield } \\
\text { (g) }\end{array}$ & $\begin{array}{c}\text { Harves } \\
t \text { index } \\
(\%)\end{array}$ & $\begin{array}{c}\text { Test } \\
\text { weight } \\
\text { (g) }\end{array}$ & $\begin{array}{l}\text { Grain } \\
\text { yield/ } \\
\text { plant } \\
(\mathrm{cm})\end{array}$ \\
\hline $\begin{array}{c}\text { Days to } \\
50 \% \\
\text { flowering }\end{array}$ & 1 & $0.988 * *$ & 0.4129 & $\begin{array}{c}0.415 \\
1\end{array}$ & -0.0343 & -0.4647 & 0.0986 & 0.1431 & 0.2543 & 0.0514 & $-0.286 * *$ & -0.1417 & 0.0805 & $-0.416^{* *}$ & 0.0493 \\
\hline $\begin{array}{l}\text { Days to } \\
\text { maturity }\end{array}$ & & 1 & 0.4238 & $\begin{array}{c}0.398 \\
7\end{array}$ & -0.0613 & -0.4721 & 0.0712 & 0.1147 & 0.1655 & 0.0592 & $-0.304 * *$ & -0.1556 & 0.1138 & $-0.385 * *$ & 0.1051 \\
\hline $\begin{array}{c}\text { Plant } \\
\text { height }(\mathrm{cm})\end{array}$ & & & 1 & 0.547 & 0.2209 & -0.4763 & -0.2092 & 0.0202 & 0.2565 & 0.1092 & -0.0621 & $0.251^{*}$ & $0 .-\overline{0}$ & 0.133 & $-0.202 *$ \\
\hline No of leafs & & & & 1 & -0.1491 & -0.2248 & -0.1404 & -0.0426 & 0.482 & 0.1318 & $0.263 * *$ & $0.314 * *$ & $0 .-\bar{c}$ & -0.0684 & -0.1488 \\
\hline $\begin{array}{l}\text { Flag leaf } \\
\text { length }(\mathrm{cm})\end{array}$ & & & & & 1 & 0.1793 & 0.2915 & -0.0464 & 0.2226 & $0.326 * *$ & $0.464 * *$ & 0.1672 & -0.1503 & $0.430 * *$ & 0.0264 \\
\hline $\begin{array}{c}\text { Flag leaf } \\
\text { width }(\mathrm{cm})\end{array}$ & & & & & & 1 & 0.2828 & -0.0602 & 0.5457 & 0.0972 & $0.256^{* *}$ & $0.286^{* *}$ & $-0.232 *$ & 0.156 & -0.0501 \\
\hline $\begin{array}{c}\text { Ear head } \\
\text { length }(\mathrm{cm})\end{array}$ & & & & & & & 1 & $0.717 * *$ & 0.1887 & 0.1378 & $0.320 * *$ & 0.1326 & $0.292 * *$ & -0.1061 & $-0.210 *$ \\
\hline $\begin{array}{c}\text { Finger } \\
\text { length }(\mathrm{cm})\end{array}$ & & & & & & & & 1 & 0.0626 & 0.1028 & 0.0907 & -0.1358 & -0.1367 & -0.1206 & $\begin{array}{c}- \\
0.358 * \\
*\end{array}$ \\
\hline $\begin{array}{c}\text { Finger } \\
\text { width }(\mathrm{cm})\end{array}$ & & & & & & & & & 1 & -0.0504 & $0.425^{* *}$ & $0.414 * *$ & 0.420 *** & $-0.288 * *$ & $\begin{array}{c}- \\
0.233^{*} \\
*\end{array}$ \\
\hline $\begin{array}{c}\text { No of } \\
\text { fingers/ ear }\end{array}$ & & & & & & & & & & 1 & -0.1401 & 0.1665 & -0.1895 & $0.415^{* *}$ & 0.0216 \\
\hline $\begin{array}{c}\text { No of } \\
\text { productive } \\
\text { tillers }\end{array}$ & & & & & & & & & & & 1 & $0.345^{* *}$ & $\begin{array}{c}- \\
0.314 * *\end{array}$ & 0.0471 & -0.0917 \\
\hline $\begin{array}{c}\text { Biological } \\
\text { yield }(\mathrm{g})\end{array}$ & & & & & & & & & & & & 1 & $\begin{array}{c}- \\
0.858^{* * *}\end{array}$ & 0.183 & -0.0871 \\
\hline $\begin{array}{c}\text { Harvest } \\
\text { index }(\%)\end{array}$ & & & & & & & & & & & & & 1 & $-0.242 *$ & $\begin{array}{c}0.565 * \\
*\end{array}$ \\
\hline $\begin{array}{c}\text { Test } \\
\text { weight }(\mathrm{g})\end{array}$ & & & & & & & & & & & & & & 1 & $-0.200 *$ \\
\hline
\end{tabular}


Table 4: Phenotypic correlation coefficient between yield and its related traits in 15 quantitative parameters in finger millet

\begin{tabular}{|c|c|c|c|c|c|c|c|c|c|c|c|c|c|c|c|}
\hline characters & $\begin{array}{c}\text { Days to } \\
50 \% \\
\text { flowering }\end{array}$ & $\begin{array}{c}\text { Days to } \\
\text { maturit } \\
y\end{array}$ & $\begin{array}{c}\text { Plant } \\
\text { height } \\
\text { (cm) }\end{array}$ & $\begin{array}{l}\text { No of } \\
\text { leafs }\end{array}$ & $\begin{array}{c}\begin{array}{c}\text { Flag } \\
\text { leaf } \\
\text { length } \\
(\mathrm{cm})\end{array} \\
\end{array}$ & \begin{tabular}{|c|}
$\begin{array}{c}\text { Flag } \\
\text { leaf } \\
\text { width } \\
(\mathrm{cm})\end{array}$ \\
\end{tabular} & $\begin{array}{c}\text { Earhead } \\
\text { length } \\
(\mathrm{cm})\end{array}$ & $\begin{array}{c}\text { Finger } \\
\text { length } \\
(\mathrm{cm})\end{array}$ & $\begin{array}{c}\text { Finger } \\
\text { width } \\
\text { (cm) }\end{array}$ & $\begin{array}{l}\text { No of } \\
\text { fingers } \\
\text { per ear }\end{array}$ & $\begin{array}{c}\text { No of } \\
\text { productive } \\
\text { tillers }\end{array}$ & $\begin{array}{c}\text { Biologic } \\
\text { al yield } \\
\text { (g) }\end{array}$ & $\begin{array}{c}\text { Harvest } \\
\text { index } \\
(\%)\end{array}$ & $\begin{array}{c}\text { Test } \\
\text { weight } \\
\text { (g) }\end{array}$ & $\begin{array}{c}\text { Grain } \\
\text { yield per } \\
\text { plant }(g)\end{array}$ \\
\hline $\begin{array}{c}\text { Days to } \\
50 \% \\
\text { flowering }\end{array}$ & 1 & $\begin{array}{c}0.9837 \\
* *\end{array}$ & $\begin{array}{c}0.2949 \\
* *\end{array}$ & $\begin{array}{c}0.297 \\
6 * *\end{array}$ & -0.0243 & $\mid \begin{array}{c}-0.2190 \\
*\end{array}$ & 0.0709 & 0.0972 & 0.1836 & 0.0532 & $-0.1969 *$ & -0.1349 & 0.0739 & $\begin{array}{c}-0.3340 \\
* *\end{array}$ & 0.0455 \\
\hline $\begin{array}{l}\text { Days to } \\
\text { maturity }\end{array}$ & & 1 & $\begin{array}{c}0.2975 \\
* *\end{array}$ & \begin{tabular}{|c|}
0.287 \\
$8 * *$ \\
\end{tabular} & -0.0363 & $\begin{array}{c}-0.2192 \\
*\end{array}$ & 0.0538 & 0.076 & 0.123 & 0.0497 & $-0.2059 *$ & -0.1483 & 0.1095 & $\begin{array}{c}-0.3079 \\
* *\end{array}$ & 0.0972 \\
\hline \begin{tabular}{|c|} 
Plant \\
height $(\mathrm{cm})$ \\
\end{tabular} & & & 1 & \begin{tabular}{|c|}
0.333 \\
$0 * *$ \\
\end{tabular} & 0.1666 & $\begin{array}{c}-0.2454 \\
*\end{array}$ & -0.0979 & 0.1113 & 0.083 & 0.0309 & -0.0309 & $0.2133 *$ & $-0.2057 *$ & 0.1263 & -0.0506 \\
\hline No of leafs & & & & 1 & -0.0925 & -0.1185 & -0.0279 & -0.0241 & $\begin{array}{c}0.2109 \\
*\end{array}$ & 0.1467 & 0.0913 & $0.2405 *$ & $-0.2399 *$ & -0.0203 & -0.0631 \\
\hline $\begin{array}{c}\text { Flag leaf } \\
\text { length }(\mathrm{cm})\end{array}$ & & & & & 1 & 0.0854 & $0.2168 *$ & -0.0117 & 0.1256 & $0.2474 *$ & $0.2983 * *$ & 0.0761 & -0.0599 & 0.1534 & 0.0165 \\
\hline \begin{tabular}{|c|} 
Flag leaf \\
width $(\mathrm{cm})$ \\
\end{tabular} & & & & & & 1 & 0.0974 & -0.0051 & $\begin{array}{c}0.2581 \\
* *\end{array}$ & -0.0063 & 0.1341 & 0.1411 & -0.1081 & 0.0468 & 0.0023 \\
\hline \begin{tabular}{|c|} 
Ear head \\
length $(\mathrm{cm})$
\end{tabular} & & & & & & & 1 & $\begin{array}{c}0.5723 \\
* *\end{array}$ & 0.1168 & 0.1355 & $0.2110 *$ & 0.0781 & -0.1619 & -0.0702 & -0.0991 \\
\hline $\begin{array}{c}\text { Finger } \\
\text { length }(\mathrm{cm})\end{array}$ & & & & & & & & 1 & -0.0338 & 0.07 & 0.0088 & -0.126 & -0.0525 & -0.0015 & -0.1888 \\
\hline \begin{tabular}{|c|} 
Finger \\
width $(\mathrm{cm})$ \\
\end{tabular} & & & & & & & & & 1 & -0.0087 & 0.1815 & $\begin{array}{c}0.2656 \\
* *\end{array}$ & $\begin{array}{c}-0.2696 \\
* *\end{array}$ & $-0.2194 *$ & -0.1287 \\
\hline \begin{tabular}{|c|} 
No of \\
fingers/ ear
\end{tabular} & & & & & & & & & & 1 & -0.0024 & 0.1549 & -0.1614 & $0.2950 * *$ & 0.0097 \\
\hline $\begin{array}{c}\text { No of } \\
\text { productive } \\
\text { tillers }\end{array}$ & & & & & & & & & & & 1 & $0.2443 *$ & $\begin{array}{c}-0.2679 \\
* *\end{array}$ & -0.0102 & -0.1642 \\
\hline \begin{tabular}{|c|} 
Biological \\
yield $(\mathrm{g})$
\end{tabular} & & & & & & & & & & & & 1 & $\begin{array}{c}-0.8134 \\
* *\end{array}$ & 0.1384 & -0.0416 \\
\hline $\begin{array}{c}\text { Harvest } \\
\text { index }(\%)\end{array}$ & & & & & & & & & & & & & 1 & -0.1304 & $0.587 * *$ \\
\hline \begin{tabular}{|c|} 
Test \\
weight $(\mathrm{g})$ \\
\end{tabular} & & & & & & & & & & & & & & 1 & -0.0649 \\
\hline \begin{tabular}{|c|}
$\begin{array}{c}\text { Grain yield } \\
\text { per plant } \\
(\mathrm{g})\end{array}$ \\
\end{tabular} & & & & & & & & & & & & & & & 1 \\
\hline
\end{tabular}

$*=$ Significane at $5 \%$ level of significance $* *=$ Significance at $1 \%$ level of signifance

Table 5: Direct and indirect effect of genotypic path coefficient for 15 characters in finger millet

\begin{tabular}{|c|c|c|c|c|c|c|c|c|c|c|c|c|c|c|c|}
\hline characters & \begin{tabular}{|c|} 
Days to \\
$50 \%$ \\
flowerin \\
$\mathrm{g}$
\end{tabular} & \begin{tabular}{|c} 
Days to \\
maturit \\
$y$
\end{tabular} & \begin{tabular}{|c|c|} 
o & Plant \\
theight \\
$(\mathbf{c m})$
\end{tabular} & $\begin{array}{l}\text { No of } \\
\text { leafs }\end{array}$ & $\begin{array}{c}\text { Flag leaf } \\
\text { length } \\
(\mathrm{cm})\end{array}$ & $\begin{array}{c}\text { Flag leaf } \\
\text { width } \\
(\mathrm{cm})\end{array}$ & $\begin{array}{c}\text { Ear head } \\
\text { length } \\
(\mathrm{cm})\end{array}$ & \begin{tabular}{|c|} 
Finger \\
length \\
$(\mathrm{cm})$
\end{tabular} & $\begin{array}{c}\text { Finge } \\
\mathbf{r} \\
\text { width } \\
\text { (cm) }\end{array}$ & $\begin{array}{c}\text { No of } \\
\text { fingers } \\
\text { per ear }\end{array}$ & $\begin{array}{c}\text { No of } \\
\text { productiv } \\
\text { e tillers }\end{array}$ & \begin{tabular}{|c|} 
Biologic \\
al \\
yield $(g)$
\end{tabular} & $c \mid \begin{array}{c}\text { Harve } \\
\text { st } \\
\text { index } \\
(\%)\end{array}$ & \begin{tabular}{|c|} 
Test \\
weight \\
$(\mathrm{g})$
\end{tabular} & \begin{tabular}{|c|} 
Grain \\
yield \\
per \\
plant $(\mathrm{g})$
\end{tabular} \\
\hline $\begin{array}{c}\text { Days to } \\
50 \% \\
\text { flowering }\end{array}$ & 1.2474 & -0.8445 & 50.2604 & \begin{tabular}{|c|}
- \\
0.411 \\
8
\end{tabular} & 0.0456 & 0.1501 & 0.0805 & -0.1168 & $\begin{array}{c}0.101 \\
5\end{array} \mid$ & 0.0312 & -0.2587 & -0.1798 & $\left|\begin{array}{c}0.163 \\
1\end{array}\right|$ & -0.2187 & 0.0493 \\
\hline $\begin{array}{l}\text { Days to } \\
\text { maturity }\end{array}$ & 1.2325 & -0.8547 & 0.2673 & $\begin{array}{c}- \\
0.395 \\
5\end{array}$ & 0.0814 & 0.1525 & 0.0581 & -0.0936 & $\left|\begin{array}{c}0.066 \\
1\end{array}\right|$ & 0.0359 & -0.2754 & -0.1973 & $\left|\begin{array}{c}0.230 \\
4\end{array}\right|$ & -0.2026 & 0.1051 \\
\hline $\begin{array}{c}\text { Plant } \\
\text { height }(\mathrm{cm})\end{array}$ & 0.515 & -0.3622 & 0.6307 & $\begin{array}{c}- \\
0.542 \\
7\end{array}$ & -0.2935 & 0.1538 & -0.1707 & -0.0165 & $\begin{array}{c}0.102 \\
4\end{array} \mid$ & 0.0663 & -0.0563 & 0.3178 & $\mid \begin{array}{c}- \\
0.615 \\
7\end{array}$ & 0.07 & -0.2015 \\
\hline No of leafs & 0.5178 & -0.3408 & 0.345 & \begin{tabular}{|c|}
- \\
0.992 \\
1 \\
\end{tabular} & 0.1981 & 0.0726 & -0.1146 & 0.0348 & $\left|\begin{array}{c}0.192 \\
5\end{array}\right|$ & 0.08 & 0.2378 & 0.3988 & \begin{tabular}{|c|}
- \\
0.742 \\
7 \\
\end{tabular} & -0.036 & -0.1488 \\
\hline $\begin{array}{c}\text { Flag leaf } \\
\text { length }(\mathrm{cm})\end{array}$ & -0.0428 & 0.0524 & 0.1393 & $\begin{array}{c}0.147 \\
9\end{array}$ & -0.9327 & -0.0579 & 0.2378 & 0.0379 & $\begin{array}{c}0.088 \\
9\end{array}$ & 0.1978 & 0.4203 & 0.2121 & $\begin{array}{c}- \\
0.304 \\
4\end{array}$ & 0.2261 & 0.0264 \\
\hline $\begin{array}{c}\text { Flag leaf } \\
\text { width }(\mathrm{cm})\end{array}$ & -0.5797 & 0.4035 & $\begin{array}{c}- \\
0.3004\end{array}$ & 0.223 & -0.2383 & -0.323 & 0.2307 & 0.0492 & $\begin{array}{c}0.217 \\
9\end{array} \mid$ & 0.059 & 0.2318 & 0.3629 & $\begin{array}{c}- \\
0.468 \\
9\end{array}$ & 0.0821 & -0.0501 \\
\hline \begin{tabular}{|c|} 
Ear head \\
length $(\mathrm{cm})$
\end{tabular} & 0.123 & -0.0609 & $\begin{array}{c}- \\
0.1319 \\
\end{array}$ & \begin{tabular}{|c|}
0.139 \\
3 \\
\end{tabular} & -0.3874 & -0.0913 & 0.8157 & -0.5851 & \begin{tabular}{|c|}
0.075 \\
4
\end{tabular} & 0.0836 & 0.2898 & 0.1682 & -0.592 & -0.0558 & -0.2095 \\
\hline $\begin{array}{c}\text { Finger } \\
\text { length }(\mathrm{cm})\end{array}$ & 0.1785 & -0.098 & 0.0127 & $\begin{array}{c}0.042 \\
3\end{array}$ & 0.0617 & 0.0195 & 0.5846 & -0.8164 & 0.025 & 0.0624 & 0.0821 & -0.1722 & $\begin{array}{c}- \\
0.276 \\
8\end{array}$ & -0.0635 & -0.358 \\
\hline $\begin{array}{c}\text { Finger } \\
\text { width }(\mathrm{cm})\end{array}$ & 0.3172 & -0.1414 & 0.1618 & \begin{tabular}{|c|}
- \\
0.478 \\
2
\end{tabular} & -0.2959 & -0.1762 & 0.1539 & -0.0511 & $\left|\begin{array}{c}0.399 \\
3\end{array}\right|$ & -0.0306 & 0.385 & 0.5246 & \begin{tabular}{|c|}
- \\
0.849 \\
9
\end{tabular} & -0.1514 & -0.2329 \\
\hline No of & 0.0641 & -0.0506 & 0.0689 & - & -0.4331 & -0.0314 & 0.1124 & -0.084 & - & 0.6069 & -0.1269 & 0.2113 & - & 0.2185 & 0.0216 \\
\hline
\end{tabular}




\begin{tabular}{|c|c|c|c|c|c|c|c|c|c|c|c|c|c|c|c|}
\hline fingers/ ear & & & & \begin{tabular}{|c|}
0.130 \\
8 \\
\end{tabular} & & & & & \begin{tabular}{|c|}
0.020 \\
1
\end{tabular} & & & & $\begin{array}{c}0.383 \\
7\end{array}$ & & \\
\hline $\begin{array}{c}\text { No of } \\
\text { productive } \\
\text { tillers }\end{array}$ & -0.3563 & 0.2598 & $\begin{array}{c}- \\
0.0392\end{array}$ & $\begin{array}{c}- \\
0.260 \\
4 \\
\end{array}$ & -0.6167 & -0.0826 & 0.261 & -0.074 & $\begin{array}{c}0.169 \\
7\end{array}$ & -0.085 & 0.9058 & 0.4381 & $\begin{array}{c}- \\
0.636 \\
7 \\
\end{array}$ & 0.0248 & -0.0917 \\
\hline $\begin{array}{c}\text { Biological } \\
\text { yield }(\mathrm{g})\end{array}$ & -0.1768 & 0.133 & 0.158 & $\begin{array}{c}- \\
0.311 \\
9\end{array}$ & -0.2222 & -0.0924 & 0.1082 & 0.1108 & $\begin{array}{c}0.165 \\
1\end{array}$ & 0.1011 & 0.3128 & 1.2687 & $\begin{array}{c}- \\
1.737 \\
8\end{array}$ & 0.0963 & -0.0871 \\
\hline $\begin{array}{c}\text { Harvest } \\
\text { index }(\%)\end{array}$ & 0.1005 & -0.0973 & $\begin{array}{c}- \\
0.1917\end{array}$ & $\begin{array}{c}0.363 \\
8\end{array}$ & 0.1997 & 0.0748 & -0.2385 & 0.1116 & $\begin{array}{c}- \\
0.167 \\
6\end{array} \mid$ & -0.115 & -0.2848 & -0.9998 & $\begin{array}{c}2.025 \\
2\end{array}$ & -0.1276 & 0.5645 \\
\hline $\begin{array}{c}\text { Test } \\
\text { weight }(\mathrm{g})\end{array}$ & -0.5184 & 0.329 & 0.0839 & $\begin{array}{c}0.067 \\
9\end{array}$ & -0.571 & -0.0504 & -0.0865 & 0.0984 & \begin{tabular}{|c|}
- \\
0.114 \\
8
\end{tabular} & 0.252 & 0.0426 & 0.2322 & -0.491 & 0.5264 & -0.1998 \\
\hline
\end{tabular}

Table 6: Direct and indirect effect of phenotypic path coefficient for 15 characters in finger millet

\begin{tabular}{|c|c|c|c|c|c|c|c|c|c|c|c|c|c|c|c|}
\hline characters & \begin{tabular}{|c|} 
Days to \\
$50 \%$ \\
flowerin \\
g \\
\end{tabular} & \begin{tabular}{|c} 
Days to \\
maturit \\
$\mathbf{y}$ \\
\end{tabular} & $\begin{array}{c}\text { Plant } \\
\text { height } \\
(\mathbf{c m})\end{array}$ & $\begin{array}{l}\text { No of } \\
\text { leafs }\end{array}$ & \begin{tabular}{|c|}
$\begin{array}{c}\text { Flag } \\
\text { leaf } \\
\text { length } \\
(\mathrm{cm})\end{array}$ \\
\end{tabular} & $\begin{array}{c}\text { Flag leaf } \\
\text { width } \\
(\mathrm{cm})\end{array}$ & $\begin{array}{c}\text { Ear head } \\
\text { length } \\
(\mathrm{cm})\end{array}$ & $\begin{array}{c}\text { Finger } \\
\text { length } \\
(\mathrm{cm})\end{array}$ & $\begin{array}{c}\text { Finger } \\
\text { width } \\
(\mathbf{c m})\end{array}$ & $\begin{array}{c}\text { No of } \\
\text { fingers } \\
\text { per ear }\end{array}$ & $\begin{array}{c}\text { No of } \\
\text { productiv } \\
\text { e tillers }\end{array}$ & \begin{tabular}{|c|} 
Biologi \\
cal \\
yield \\
$(\mathrm{g})$ \\
\end{tabular} & $\begin{array}{c}\text { Harve } \\
\text { st } \\
\text { index } \\
(\%)\end{array}$ & \begin{tabular}{|c|} 
Test \\
weig \\
ht \\
$(\mathrm{g})$ \\
\end{tabular} & $\begin{array}{c}\text { Grain } \\
\text { yield per } \\
\text { plant }(g)\end{array}$ \\
\hline $\begin{array}{c}\text { Days to } \\
50 \% \\
\text { flowering }\end{array}$ & -0.2976 & 0.3906 & -0.0067 & $\begin{array}{c}0.000 \\
5\end{array}$ & -0.0006 & -0.005 & 0.002 & 0.0042 & $\begin{array}{c}- \\
0.0084\end{array}$ & 0.0034 & 0.0073 & -0.1786 & 0.1206 & $\begin{array}{c}0.01 \\
39\end{array}$ & 0.0455 \\
\hline $\begin{array}{l}\text { Days to } \\
\text { maturity }\end{array}$ & -0.2927 & 0.3971 & -0.0068 & $\begin{array}{c}0.000 \\
5\end{array}$ & -0.0009 & -0.005 & 0.0015 & 0.0033 & $\begin{array}{c}- \\
0.0056 \\
\end{array}$ & 0.0032 & 0.0076 & -0.1964 & 0.1787 & \begin{tabular}{|c|}
0.01 \\
28 \\
\end{tabular} & 0.0972 \\
\hline $\begin{array}{c}\text { Plant } \\
\text { height }(\mathrm{cm})\end{array}$ & -0.0878 & 0.1181 & -0.0229 & $\begin{array}{c}0.000 \\
5\end{array}$ & 0.0041 & -0.0056 & -0.0028 & 0.0048 & $\begin{array}{c}- \\
0.0038\end{array}$ & 0.002 & 0.0011 & 0.2825 & $\begin{array}{c}- \\
0.3356\end{array}$ & $\begin{array}{c}- \\
0.00 \\
52\end{array}$ & -0.0506 \\
\hline No of leafs & -0.0886 & 0.1143 & -0.0076 & $\begin{array}{c}0.001 \\
6\end{array}$ & -0.0023 & -0.0027 & -0.0008 & -0.001 & $\begin{array}{c}- \\
0.0097 \\
\end{array}$ & 0.0093 & -0.0034 & 0.3184 & $\begin{array}{c}- \\
0.3915 \\
\end{array}$ & \begin{tabular}{|c|}
0.00 \\
08 \\
\end{tabular} & -0.0631 \\
\hline $\begin{array}{c}\text { Flag leaf } \\
\text { length }(\mathrm{cm})\end{array}$ & 0.0072 & -0.0144 & -0.0038 & $\begin{array}{c}- \\
0.000 \\
1 \\
\end{array}$ & 0.0244 & 0.0019 & 0.0062 & -0.0005 & $\begin{array}{c}- \\
0.0057\end{array}$ & 0.0157 & -0.011 & 0.1008 & $\begin{array}{c}- \\
0.0977 \\
\end{array}$ & \begin{tabular}{|c|}
- \\
0.00 \\
64 \\
\end{tabular} & 0.0165 \\
\hline $\begin{array}{c}\text { Flag leaf } \\
\text { width }(\mathrm{cm})\end{array}$ & 0.0652 & -0.087 & 0.0056 & \begin{tabular}{|c|}
- \\
0.000 \\
2
\end{tabular} & 0.0021 & 0.0228 & 0.0028 & -0.0002 & $\begin{array}{c}- \\
0.0118\end{array}$ & -0.0004 & -0.0049 & 0.1868 & $\begin{array}{c}- \\
0.1764\end{array}$ & \begin{tabular}{|c|}
- \\
0.00 \\
19
\end{tabular} & 0.0023 \\
\hline $\begin{array}{c}\text { Ear head } \\
\text { length }(\mathrm{cm})\end{array}$ & -0.0211 & 0.0214 & 0.0022 & $\begin{array}{c}0.001 \\
1\end{array}$ & 0.0053 & 0.0022 & 0.0287 & 0.0247 & $\begin{array}{c}- \\
0.0053 \\
\end{array}$ & 0.0086 & -0.0078 & 0.1034 & $\begin{array}{c}- \\
0.2641 \\
\end{array}$ & \begin{tabular}{|c|}
0.00 \\
29 \\
\end{tabular} & -0.0991 \\
\hline $\begin{array}{c}\text { Finger } \\
\text { length }(\mathrm{cm})\end{array}$ & -0.0289 & 0.0302 & -0.0025 & $\begin{array}{c}0.001 \\
1\end{array}$ & -0.0003 & -0.0001 & 0.0164 & 0.0431 & 0.0015 & 0.0044 & -0.0003 & -0.1668 & \begin{tabular}{|c|}
- \\
0.0856 \\
\end{tabular} & \begin{tabular}{|c|}
0.00 \\
01
\end{tabular} & -0.1888 \\
\hline $\begin{array}{c}\text { Finger } \\
\text { width }(\mathrm{cm})\end{array}$ & -0.0546 & 0.0489 & -0.0019 & $\begin{array}{c}0.000 \\
3 \\
\end{array}$ & 0.0031 & 0.0059 & 0.0034 & -0.0015 & \begin{tabular}{c|}
- \\
0.0458 \\
\end{tabular} & -0.0006 & -0.0067 & 0.3517 & $\begin{array}{c}- \\
0.4399 \\
\end{array}$ & $\begin{array}{c}0.00 \\
91 \\
\end{array}$ & -0.1287 \\
\hline $\begin{array}{c}\text { No of } \\
\text { fingers/ ear }\end{array}$ & -0.0158 & 0.0197 & -0.0007 & $\left|\begin{array}{c}0.000 \\
2\end{array}\right|$ & 0.006 & -0.0001 & 0.0039 & 0.003 & 0.0004 & 0.0635 & 0.0001 & 0.205 & $\begin{array}{c}- \\
0.2633\end{array}$ & $\begin{array}{c}- \\
0.01 \\
22 \\
\end{array}$ & 0.0097 \\
\hline $\begin{array}{c}\text { No of } \\
\text { productive } \\
\text { tillers }\end{array}$ & 0.0586 & -0.0818 & 0.0007 & $\left|\begin{array}{c}0.000 \\
1\end{array}\right|$ & 0.0073 & 0.0031 & 0.0061 & 0.0004 & $\begin{array}{c}- \\
0.0083\end{array}$ & -0.0002 & -0.0369 & 0.3234 & $\begin{array}{c}- \\
0.4372\end{array}$ & $\begin{array}{c}0.00 \\
04\end{array}$ & -0.1642 \\
\hline $\begin{array}{c}\text { Biological } \\
\text { yield }(\mathrm{g})\end{array}$ & 0.0401 & -0.0589 & -0.0049 & $\begin{array}{c}0.000 \\
4\end{array} \mid$ & 0.0019 & 0.0032 & 0.0022 & -0.0054 & $\mid \begin{array}{c}- \\
0.0122\end{array}$ & 0.0098 & -0.009 & 1.324 & $\begin{array}{c}- \\
1.3271\end{array}$ & \begin{tabular}{|c|}
- \\
0.00 \\
57 \\
\end{tabular} & -0.0416 \\
\hline $\begin{array}{c}\text { Harvest } \\
\text { index }(\%)\end{array}$ & -0.022 & 0.0435 & 0.0047 & $\left|\begin{array}{c}- \\
0.000 \\
4\end{array}\right|$ & -0.0015 & -0.0025 & -0.0046 & -0.0023 & 0.0123 & -0.0102 & 0.0099 & -0.9643 & 1.6316 & $\begin{array}{c}0.00 \\
54\end{array}$ & 0.587 \\
\hline $\begin{array}{c}\text { Test } \\
\text { weight }(\mathrm{g})\end{array}$ & 0.0994 & -0.1223 & -0.0029 & $\begin{array}{c}0.000 \\
1\end{array}$ & 0.0037 & 0.0011 & -0.002 & -0.0001 & 0.01 & 0.0187 & 0.0004 & 0.1832 & $\begin{array}{c}- \\
0.2128\end{array}$ & $\begin{array}{c}- \\
0.04 \\
15\end{array}$ & -0.0649 \\
\hline
\end{tabular}

Research Guide or Chairperson of research: Prof. (Dr.)

\section{Suresh B.G}

University: Sam Higginbottom University of Agriculture Technology and Sciences, Prayagraj, Uttar Pradesh, India Research project name: Correlation and Path analysis Studies in Finger Millet for Yield and Yield Contributing Traits [Eleusine coracana (L.) Gaertn.]

\section{References}

1. Al- Jibouri HA, Muller PA, Robinson HF. Genetic and environmental variances and co-variances in upland cotton cross of inter specific origin. Journal of Agronomy 1958;50:633-636.

2. Ashok Singamsetti, Patro TSSK, Anuradha N, Divya M. Studies on genetic variability for yield and yield attributing traits in Finger millet (Eluesine coracana L. Gaertn). International Journal of Current Microbiology and Applied Sciences 2018;7:90-95.

3. Burton GW. Quantitative inheritance in grasses. Pro VI International Grassland Congress, 1952, 277-283. 
4. Burton GW, Devane EH. Estimating heritability in tall fescue (Festuca arundinaceae) from replicated clonal material 1. Agronomy Journal 1953;45(10):478-481.

5. Chavan BR, LN Jawale TA, Shinde AV. Studies on Genetic Variability for Yield and Yield Contributing Traits in Finger Millet Eleusine coracana (L.) Gaertn. International journal of current microbiology and applied Sciences 2020;8(09):2276-2281.

6. Chennaveeraiah MS, Hieremanth SC. Genome analysis of Eleusine coracana (L.) Gaertn. Euphytica 1974;23(3):489-495.

7. Devaliya DS, Singh M, Intawala CG. Genetic Variability Studies in Finger millet [Eleusine coracana (L.) Gaertn.]. International Journal of Current Microbiology and Applied Sciences 2017;6(1):1007-1011.

8. Dewey DR, Lu KH. Correlation path coefficient analysis of components of crested Wheat grass, Agronomy Journal 1959;51:513-518.

9. Fisher RA, Yates. Statistical method for research workers. Oliver and Boyd Ltd. Edinburghand London, 1968, 10.

10. Gohel DS, Chaudhari SB. Study of correlation and path analysis of finger millet genotypes (Eleusine coracana $\mathrm{L}$. Gaertn). Journal of Pharmacognosy and Phytochemistry 2018;7(6):1283-1288.

11. Hilu KW, De Wet JMJ. Domestication of Eleusine coracana. Economic botany 1976;30(3):199-208.

12. Johnson HW, Robinson HF, Comstock R. Estimates of genetic and environmental variability in Soyabeans 1. Agronomy Journal 1955;47(7):314-318.

13. Jansen PMC, Ong HC. Eluesine coracana L. Gaertn ev. Group Finger millet. Plant resources of South-East Asia No 10. Cereals, Backhuys publishers, Leiden, Netherlands, 1996, 90-95.

14. Lule D, Tesfaye K, Fetene M, De Villiers S. Inheritance and association of quantitative traits in finger millet (Eleusine coracana Subsp. Coracana) landraces collected from Eastern and South Eastern Africa. International Journal of Journal of Genetics 2012;2(2):12-21.

15. Lush JL. Heritability of quantitative characters in farm animals. Heritability of quantitative characters in farm animals, 1949.

16. Negi AS, Pandey PK, Shotria PK. Correlation and Path Coefficient Analysis of Component Attributes in Finger Millet (Eleusine coracana (L.) Gaertn). Environment \& Ecology 2016;34(4C):1852-1856.

17. Negi S, Bhatt A, Kumar V. Character association and path analysis for yield and its related traits in finger millet (Eleusine coracana (L.) Gaertn) genotypes. Journal of Applied and Natural Science 2017;9(3):16241629.

18. Purseglove JW. Tropical crops: Monocotyledons. Molecular Nutrition and Food research 1972;19(56):395-524 